\title{
On the Error Terms of Chebyshev Functions for SL4
}

\author{
DŽENAN GUŠIĆ \\ University of Sarajevo \\ Faculty of Sciences and Mathematics \\ Department of Mathematics \\ Zmaja od Bosne 33-35, 71000 Sarajevo \\ BOSNIA AND HERZEGOVINA \\ dzenang@pmf.unsa.ba
}

\begin{abstract}
Our object of research are certain higher order counting functions of Chebyshev type, associated to the compact symmetric space SL4. In particular, we consider the function $\psi_{1}(x)$ resp. $\psi_{3}(x)$, of order 1 resp. 3. As it is well known, any such function can be represented as a sum of some explicit part, and the corresponding error term. The explicit part is usually indexed over singularities of the attached Selberg zeta functions, while the error term depends on the dimension of the underlying symmetric space. Thus, these functions generalize the classical yes function $\pi(x)$ counting prime geodesics of appropriate length. More precisely, the Chebyshev functions divided by adequate power of $x$, represent quite natural approximations for the function $\pi(x)$. In this research, we are particularly interested in the error terms of $\psi_{1}(x) / x$ and $\psi_{3}(x) / x^{3}$.
\end{abstract}

Key-Words: Chebyshev functions, orders of functions, weighted theorems, counting functions, error terms Received: September 14, 2019. Revised: February 19, 2020. Accepted: March 10, 2020. Published: March 27, 2020.

\section{Introduction}

Recently, we proved that in the case of compact symmetric spaces obtained as quotients of the Lie group $S L_{4}(\mathbb{R})$, the corresponding length spectrum $\pi(x)$ is given as the sum of the explicit part 2 li $(x)$, and the remainder $O\left(x^{1-\frac{1}{2 D}}(\log x)^{-1}\right)$ during the process $x$ $\rightarrow+\infty$, where $\pi(x)$ is a yes function counting prime geodesics of the length not larger than $\log x, \operatorname{li}(x)=$ $\int_{2}^{x} \frac{d t}{\log t}$ is the integral logarithm, and $D$ is the degree of the polynomial that appears in the functional equation of the corresponding Selberg zeta function.

It is well known that the length spectrum stated above easily follows from the corresponding equation for the function $\psi_{k}(x), k \in \mathbb{N} \cup\{0\}$ (these functions are defined below). Such reasoning can be found in [23], as well as in [2]-[4], [12], [14]-[16], [25], [28], etc.

Wa also refer to [5], [17]-[19], [21], [26], [6].

Thus, for example, the length spectrum stated above follows from the fact that $\psi_{0}(x)$ is the sum of $2 x$ and $O\left(x^{1-\frac{1}{2 D}}\right)$, as $x \rightarrow+\infty$.

In other words, the knowledge about $\pi(x)$ is equivalent to the knowledge about $\psi_{0}(x)$.

Note that $\psi_{1}(x)=\int_{2}^{x} \psi_{0}(t) d t$. This means that, in some sense, the knowledge about $\psi_{0}(x)$ (and hence about $\pi(x)$ ) can be compared to the knowledge about $\frac{\psi_{1}(x)}{x}$.

However, any estimate for the function $\frac{\psi_{1}(x)}{x}$ (or more generally for the function $\frac{\psi_{k}(x)}{x^{k}}$ ) is known in literature as a generalized length spectrum (or a weighted prime geodesic theorem).

Our goal is to prove the generalized length spectrum, i.e., that $\frac{\psi_{1}(x)}{x}$ can be written as the sum of the explicit part $\sum_{I}$ and the error term $O\left(x^{\frac{1}{2}-\frac{1}{2 D}}\right)$, as $x$ $\rightarrow+\infty$, where the sum $\sum_{I}$ is indexed over singularities of the corresponding Selberg zeta functions inside $\left(\frac{1}{2}-\frac{1}{2 D}, \frac{3}{4}\right]$.

The generalized length spectrum can be also found in [1], [13], etc., and not necessarily for the same underlying symmetric space.

For the background on the representation theory of semi-simple groups, we refer to [20] (see also, [27]).

For the Selberg trace formula in this setting, see [29].

\section{Notation}

Let $k \in \mathbb{Z}$, and $I_{k}=\{0,-1, \ldots,-k\}$.

Put $I_{q}, q \in\{0,1, \ldots, 4\}$ to be the set of all $j \in I_{k}$ such that $j$ is a singularity of the Selberg zeta function $Z_{P, \bigwedge^{q} \overline{\mathfrak{n}}}\left(s+\frac{q}{4}\right)$, and $I_{q}^{\prime}=I_{k} \backslash I_{q}$. 
The set of the remaining singularities $s^{q}$ of $Z_{P, \bigwedge^{q} \overline{\mathfrak{n}}}\left(s+\frac{q}{4}\right)$ will be denoted by $S^{q}$.

Note that by Theorem 3.2.1 in [8], the function $Z_{P, \wedge^{q} \overline{\mathfrak{n}}}(s)$ extends to a meromorphic function on the whole of $\mathbb{C}$, and its singularities lie in $\mathbb{R} \cup\left(\frac{1}{2}+i \mathbb{R}\right)$ (see also, [7]).

Precise locations and the orders of the singularities of $Z_{P, \wedge^{q} \overline{\mathfrak{n}}}(s)$ are described by Proposition 3.4.3 in [8] and the discussion afterwards.

Thus, $Z_{P, 1}(s)$ has a double zero at $s=1$, and, apart from that, all poles and zeros of $Z_{P, 1}(s)$ lie in the strip $0 \leq \operatorname{Re}(s) \leq \frac{3}{4}$.

We also put $S_{\mathbb{R}}^{q}=S^{q} \cap \mathbb{R}$, and $S_{\frac{1}{2}-\frac{q}{4}}^{q}=S^{q} \backslash S_{\mathbb{R}}^{q}$.

$\overline{\mathfrak{n}}$ is the complexified Lie algebra of $\bar{N}$ (see, [24]).

$P=M A N$ is a parabolic subgroup of $G$ with Levi component $M A$ and the unipotent radical $N$.

We point out that in this paper the underlying symmetric space $X_{\Gamma}$ is given by $X_{\Gamma}=\Gamma \backslash G / K$, where $G=S L_{4}(\mathbb{R}), K$ is the maximal compact subgroup of $G$ (thus, $K=S O(4)$ ), and $\Gamma$ is a discrete, co-compact subgroup of $G$.

By $\left[24\right.$, p. 63, (3.7)], $\psi_{k}(x)$ is the sum of the residues $c_{\alpha}(q, k)$ 's at $\alpha$ 's over $q \in\{0,1, \ldots, 4\}$ and the set of poles $S_{k, q}$ of the function in the case at hand.

It is not so hard to find the residues $c_{\alpha}(q, k)$ 's depending on whether $\alpha$ 's are the elements of $S^{q}, I_{q}$, and $I_{q}^{\prime}$.

We denote by $o_{z}^{q}$ the order of the singularity $z$ of $Z_{P, \wedge^{q} \overline{\mathfrak{n}}}\left(s+\frac{q}{4}\right)$, and by $a_{i, z}^{q}$ 's the coefficients in the expansion of the logarithmic derivative $\frac{Z_{P, \wedge^{q} \overline{\mathfrak{n}}}^{\prime}\left(s+\frac{q}{4}\right)}{Z_{P, \wedge^{q}}\left(s+\frac{q}{4}\right)}$ around $z$.

We note that the counting function $\psi_{k}(x)$ is defined by $\psi_{j}(x)=\int_{0}^{x} \psi_{j-1}(t) d t, j \in \mathbb{N}$, where $\psi_{0}(x)$ is given by $\sum_{[\gamma] \in \mathcal{E}_{P}(\Gamma)} \chi_{1}\left(\Gamma_{\gamma}\right) l_{\gamma_{0}}$.

Here, $\mathcal{E}_{P}(\Gamma)$ is the set of all $\Gamma$-conjugacy classes $[\gamma]$, such that the element $\gamma \in \Gamma$ is conjugate in $G$ to an element $a_{\gamma} b_{\gamma} \in A^{-} B$, where $A^{-}$is the negative Weyl chamber in $A$ with respect to the root system given by the choice of parabolic, and $B$ is a compact Cartan subgroup of $M$.

If $\gamma$ is a conjugate to $a_{\gamma} b_{\gamma}$, we define the length $l_{\gamma}$ of $\gamma$ to be $l_{\gamma}=b\left(\log a_{\gamma}, \log b_{\gamma}\right)^{\frac{1}{2}}$, where $b$ is an invariant bilinear form on the complexified Lie algebra $\mathfrak{g}=s l_{4}(\mathbb{C})$ of $G$.

Furthermore, $\chi_{1}\left(\Gamma_{\gamma}\right)$ is the first higher Euler characteristics of the symmetric space $X_{\Gamma_{\gamma}}=\Gamma_{\gamma} \backslash$ $G_{\gamma} / K_{\gamma}$, where $G_{\gamma}$ and $\Gamma_{\gamma}$ are the centralizers of $\gamma$ in $G$ and $\Gamma$, respectively, and $K_{\gamma}=K \cap G_{\gamma}$.

In this research, we apply the known differential operator $\Delta_{k}^{+}$(see, e.g., [24]).
Furthermore, we make use of the fact that the number $N(t)$ of the vertical singularities is bounded by $O\left(t^{D}\right)$.

\section{Results}

The following theorem represents the main result of our research.

Theorem 1. Let $X_{\Gamma}$ be as above. Then,

$$
\begin{aligned}
& \frac{\psi_{1}(x)}{x} \\
= & x+\sum_{q=0}^{2}(-1)^{q} \sum_{\substack{s^{q} \in S_{\mathbb{R}}^{q} \\
\frac{1}{2}-\frac{1}{2 D}<s^{q} \leq \frac{3}{4}}} o_{s^{q}}^{q}\left(s^{q}\right)^{-1} \times \\
& \times\left(s^{q}+1\right)^{-1} x^{s^{q}}+O\left(x^{\frac{1}{2}-\frac{1}{2 D}}\right)
\end{aligned}
$$

as $x \rightarrow+\infty$.

Proof. We shall denote by $h$ the constant which will be fixed later.

For now, we shall assume that this constant is dominated by $x$.

Let $z \in S_{\frac{1}{2}-\frac{q}{4}}^{q}$.

We obtain, $h^{-(k-1)} \Delta_{k-1}^{+} c_{z}(q, k)$ is $O\left(h^{-(k-1)}|z|^{-k-1} x^{\frac{1}{2}+k}\right)$ (this estimate follows from the very definition of the differential operator $\Delta_{k-1}^{+}$).

On the other side, the integral representation of the operator yields that $h^{-(k-1)} \Delta_{k-1}^{+} c_{z}(q, k)$ is $O\left(|z|^{-2} x^{\frac{3}{2}}\right)$ as well. larities.

First, we estimate the sums over complex singu-

As it is usual, the complex singularities $z \in S_{\frac{1}{2}-\frac{q}{4}}^{q}$ are divided into two classes, those whose modulus is not smaller that $M$, and the remaining ones, where $M$ is a constant.

Hence, taking into account the number of the complex singularities, we obtain that the sum of $h^{-(m D-1)} \Delta_{m D-1}^{+} c_{z}(q, m D)$ over $q \in\{0,1, \ldots, 4\}$ and $z \in$ $S_{\frac{1}{2}-\frac{q}{4}}^{q}$ is bounded by the sum of $O\left(x^{\frac{3}{2}} M^{D-2}\right)$ and $O\left(h^{-(m D-1)} x^{\frac{1}{2}+m D} M^{D-m D-1}\right)$, where $k$ is taken to be some $m D$ for some even $m$.

Since $h^{-(k-1)} \Delta_{k-1}^{+} c_{1}(0, k)$ is $\tilde{x}^{2}$ for some $\tilde{x} \in[x, x+(k-1) h]$, it follows that $h^{-(k-1)} \Delta_{k-1}^{+} c_{1}(0, k)$ is $O\left(x^{2}\right)$. 
Having in mind the fact that $h^{-(k-1)} \Delta_{k-1}^{+} c_{1}(0, k)$ is

$$
\begin{aligned}
& 2 h^{-(k-1)} \frac{1}{(k+1) !} \sum_{i=0}^{k-1}(-1)^{i}\left(\begin{array}{c}
k-1 \\
i
\end{array}\right) \times \\
& \times \sum_{j=0}^{1+k}\left(\begin{array}{c}
1+k \\
j
\end{array}\right) x^{1+k-j}((k-1-i) h)^{j},
\end{aligned}
$$

we may write

$$
h^{-(k-1)} \Delta_{k-1}^{+} c_{1}(0, k)=P x^{2}+Q x+R
$$

for some $P, Q$ and $R$.

Thus, $P$ is

$$
\begin{aligned}
& 2 \frac{1}{(m D+1) !}\left(\begin{array}{c}
m D+1 \\
m D-1
\end{array}\right) \times \\
& \times \sum_{i=0}^{m D-1}(-1)^{i}\left(\begin{array}{c}
m D-1 \\
i
\end{array}\right)(m D-1-i)^{m D-1},
\end{aligned}
$$

i.e., $P$ is 1 . Furthermore, $Q$ is

$$
\begin{aligned}
& 2 h \frac{1}{(m D+1) !}\left(\begin{array}{c}
m D+1 \\
m D
\end{array}\right) \times \\
& \times \sum_{i=0}^{m D-1}(-1)^{i}\left(\begin{array}{c}
m D-1 \\
i
\end{array}\right)(m D-1-i)^{m D},
\end{aligned}
$$

i.e., $Q$ is $(m D-1) h$. And, finally, $R$ is given by

$$
\begin{aligned}
& 2 h^{2} \frac{1}{(m D+1) !} \times \\
& \times \sum_{i=0}^{m D-1}(-1)^{i}\left(\begin{array}{c}
m D-1 \\
i
\end{array}\right)(m D-1-i)^{m D+1} .
\end{aligned}
$$

Consequently, $h^{-(m D-1)} \Delta_{m D-1}^{+} c_{1}(0, m D)$ is the sum of $x^{2}, O(h x)$ and $O\left(h^{2}\right)$.

Now, we compare each of the error terms $O(h x)$, $O\left(h^{2}\right)$ with $O\left(x^{\frac{3}{2}} M^{D-2}\right)$ and $O\left(h^{-(m D-1)} x^{\frac{1}{2}+m D} M^{D-m D-1}\right)$.

First, we compare $O\left(h^{2}\right)$ with $O\left(x^{\frac{3}{2}} M^{D-2}\right)$ and $O\left(h^{-(m D-1)} x^{\frac{1}{2}+m D} M^{D-m D-1}\right)$.
If $h$ is $x^{\alpha}$ and $M$ is $x^{\beta}$, we establish the equalities

$$
h^{2}=x^{\frac{3}{2}} M^{D-2}=h^{-(m D-1)} x^{\frac{1}{2}+m D} M^{D-m D-1} .
$$

Thus, we solve the system

$$
\begin{aligned}
2 \alpha & =\frac{3}{2}+\beta D-2 \beta \\
& =-\alpha m D+\alpha+\frac{1}{2}+m D+\beta D-\beta m D-\beta
\end{aligned}
$$

i.e., the system

$$
\begin{aligned}
& \frac{3}{2}+\beta D-2 \beta \\
= & -\left(\frac{3}{4}+\frac{1}{2} \beta D-\beta\right) m D+\frac{3}{4}+ \\
& \frac{1}{2} \beta D-\beta+\frac{1}{2}+m D+\beta D-\beta m D-\beta .
\end{aligned}
$$

We obtain, $\beta=\frac{1}{2 D}$, and $\alpha=\frac{3}{4}+\frac{1}{2} \beta D-\beta=\frac{1}{2}$ $-\frac{1}{2 D}$, i.e., $h=x^{\frac{1}{2}-\frac{1}{2 D}}$, and $M=x^{\frac{1}{2 D}}$.

Since $h$ is dominated by $x$, it follows that our previous calculations are valid.

In this case, the dominant error term is $O(h x)$, i.e., $O\left(x^{\frac{3}{2}-\frac{1}{2 D}}\right)$.

Second, we compare $O(h x)$ and $O\left(x^{\frac{3}{2}} M^{D-2}\right)$, $O\left(h^{-(m D-1)} x^{\frac{1}{2}+m D} M^{D-m D-1}\right)$.

Hence,

$$
h x=x^{\frac{3}{2}} M^{D-2}=h^{-(m D-1)} x^{\frac{1}{2}+m D} M^{D-m D-1},
$$

i.e.,

$$
\begin{aligned}
\alpha+1= & \frac{3}{2}+\beta D-2 \beta \\
= & -\alpha m D+\alpha+\frac{1}{2}+m D+ \\
& \beta D-\beta m D-\beta,
\end{aligned}
$$

i.e.,

$$
\begin{aligned}
& \frac{3}{2}+\beta D-2 \beta \\
= & -\left(\frac{1}{2}+\beta D-2 \beta\right) m D+\frac{1}{2}+\beta D-2 \beta+ \\
& \frac{1}{2}+m D+\beta D-\beta m D-\beta .
\end{aligned}
$$


It follows that $\beta=\frac{1}{2 D-2}, \alpha=\frac{1}{2}+\beta D-2 \beta=$ $\frac{1}{2}+\frac{D}{2 D-2}-\frac{1}{D-1}$, i.e., $h=x^{\frac{1}{2}+\frac{D-2}{2 D-2}}, M=x^{\frac{1}{2 D-2}}$.

In this case, the dominant error term $O(h x)$ is $O\left(x^{\frac{3}{2}+\frac{D-2}{2 D-2}}\right)$.

Obviously, the first scenario gives a better result.

Thus, we are interested in the error term $O\left(x^{\frac{3}{2}-\frac{1}{2 D}}\right)$ achieved for the choice $h=x^{\frac{1}{2}-\frac{1}{2 D}}, M$ $=x^{\frac{1}{2 D}}$.

The sum of the elements $h^{-(k-1)} \Delta_{k-1}^{+} c_{s^{q}}(q, k)$ along $q \in\{0,1, \ldots, 4\}$ and $s^{q} \in S_{\mathbb{R}}^{q}, 0<s^{q} \leq \frac{3}{4}$ is

$$
\begin{aligned}
& \sum_{q=0}^{3}(-1)^{q} \sum_{\substack{s^{q} \in S_{\mathbb{R}}^{q} \\
0<s^{q} \leq \frac{3}{4}}} o_{s^{q}}^{q}\left(s^{q}\right)^{-1}\left(s^{q}+1\right)^{-1} x^{s^{q}+1} \\
& +O\left(x^{\frac{3}{4}} h\right) .
\end{aligned}
$$

Here, we applied the fact that

$$
\sum_{j=1}^{+\infty}\left(\begin{array}{c}
s^{q}+1 \\
j
\end{array}\right)=2^{s^{q}+1}-\left(\begin{array}{c}
s^{q}+1 \\
0
\end{array}\right)=2^{s^{q}+1}-1 .
$$

Since $h^{-(k-1)} \Delta_{k-1}^{+} c_{-j}(q, k)$ is 0 for $-j \in$ $\{-2,-3, \ldots,-k\}$, it follows that the corresponding sum over $q \in\{0,1, \ldots, 4\}$ and $-j \in\{-2,-3, \ldots,-k\}$ is equal to 0 .

Furthermore, we estimate

$$
\begin{aligned}
& \sum_{q=0}^{3}(-1)^{q} h^{-(k-1)} \Delta_{k-1}^{+} c_{-1}(q, k) \\
& +h^{-(k-1)} \Delta_{k-1}^{+} c_{-1}(4, k) .
\end{aligned}
$$

Reasoning as above, we conclude that $h^{-(k-1)} \Delta_{k-1}^{+} c_{-1}(q, k)$ is $-\frac{Z_{P, \wedge^{q} \overline{\mathfrak{n}}}^{\prime}\left(-1+\frac{q}{4}\right)}{Z_{P, \wedge^{q} \overline{\mathfrak{n}}}\left(-1+\frac{q}{4}\right)}$ for $q$ $\in\{0,1, \ldots, 3\}$.

If $-1 \in I_{4}^{\prime}$, then $h^{-(k-1)} \Delta_{k-1}^{+} c_{-1}(4, k)$ is $-\frac{Z_{P, \Lambda^{4} \overline{\mathfrak{n}}}^{\prime}(0)}{Z_{P, \Lambda^{4}}(0)}$.

Furthermore, if $-1 \in I_{4}$, then $h^{-(k-1)} \Delta_{k-1}^{+} c_{-1}(4, k)$ is $O(\log x)$.

Thus, the corresponding sum is $O(\log x)$.

Similarly,

$$
\sum_{q=0}^{4}(-1)^{q} h^{-(k-1)} \Delta_{k-1}^{+} c_{0}(q, k)=O(x \log x) .
$$

Since $h^{-(k-1)} \Delta_{k-1}^{+} c_{s}^{q}(q, k)$ is $o_{s^{q}}^{q}\left(s^{q}\right)^{-1}\left(s^{q}+1\right)^{-1} \tilde{x}_{s^{q}, q, k}^{s^{q}+1}$ for $q \in\{0,1, \ldots, 4\}, s^{q} \in$ $S_{\mathbb{R}}^{q},-1<s^{q}<0$, where $\tilde{x}_{s^{q}, q, k} \in[x, x+(k-1) h]$, it follows that the corresponding sum is $O(x)$.

Now, taking $h=x^{\frac{1}{2}-\frac{1}{2 D}}, M=x^{\frac{1}{2 D}}$, and combining the estimates derived above with the fact that $\psi_{1}(x)$ is not larger than $h^{-(k-1)} \Delta_{k-1}^{+} \psi_{k}(x)$, we obtain that $\psi_{1}(x)$ is not larger than

$$
\begin{aligned}
& x^{2}+\sum_{q=0}^{3}(-1)^{q} \sum_{\substack{s^{q} \in S_{\mathbb{R}}^{q} \\
0<s^{q} \leq \frac{3}{4}}} o_{s^{q}}^{q}\left(s^{q}\right)^{-1}\left(s^{q}+1\right)^{-1} x^{s^{q}+1} \\
& +O\left(x^{\frac{3}{2}-\frac{1}{2 D}}\right) .
\end{aligned}
$$

Similarly, one concludes that the last sum is not larger than $\psi_{1}(x)$.

This completes the proof.

\section{Additional results}

In this section we shall consider the function $\psi_{3}(x)$ in detail.

We may assume that $h$ is a constant such that $|h|$ is not larger than $C x$, where $C$ is some positive constant.

First, suppose that $z \in S_{\frac{1}{2}-\frac{q}{4}}^{q}$.

In this case, $h^{-(k-3)} \Delta_{k-3}^{+} c_{z}(q, k)$ can be estimated by

$$
O\left(h^{-(k-3)}|z|^{-k-1} x^{\frac{1}{2}+k}\right)
$$

and

$$
O\left(|z|^{-4} x^{\frac{7}{2}}\right)
$$

For a constant $M$, we shall consider the elements $z \in S_{\frac{1}{2}-\frac{q}{4}}^{q}$, such that $\left|\frac{1}{2}-\frac{q}{4}\right|<|z| \leq M$, and $|z|>$ $M$.

In the first resp. the second case, we shall apply the second resp. the first bound given above.

Having in mind the fact that $N(t)$ is estimated by $O\left(t^{D}\right)$, it easily follows that the sum 
of $h^{-(k-3)} \Delta_{k-3}^{+} c_{z}(q, k)$ (along $0 \leq q \leq 4$ and $z \in$ $\left.S_{\frac{1}{2}-\frac{q}{4}}^{q}\right)$ is

$$
O\left(x^{\frac{7}{2}} M^{D-4}\right)+O\left(h^{-(k-3)} x^{\frac{1}{2}+k} M^{D-k-1}\right) .
$$

Reasoning as earlier, we conclude that $h^{-(k-3)} \Delta_{k-3}^{+} c_{1}(0, k)$ is $\frac{1}{12}(x+\varepsilon)^{4}$, where $\varepsilon \in$ $[0,(k-3) h]$.

Hence, $h^{-(k-3)} \Delta_{k-3}^{+} c_{1}(0, k)$ is

$$
\begin{aligned}
& \frac{1}{12} x^{4} \\
& +O\left(x^{3} h\right)+O\left(x^{2} h^{2}\right)+O\left(x h^{3}\right)+O\left(h^{4}\right) \\
= & \frac{1}{12} x^{4}+O\left(x^{3} h\right) .
\end{aligned}
$$

Although we applied the fact that the error terms $O\left(x^{2} h^{2}\right), O\left(x h^{3}\right)$ and $O\left(h^{4}\right)$ are contained in $O\left(x^{3} h\right)$, we shall compare each of them with previously obtained error terms $O\left(x^{\frac{7}{2}} M^{D-4}\right)$ and $O\left(h^{-(k-3)} x^{\frac{1}{2}+k} M^{D-k-1}\right)$.

The first case: $O\left(x^{3} h\right), O\left(x^{\frac{7}{2}} M^{D-4}\right)$ and $O\left(h^{-(k-3)} x^{\frac{1}{2}+k} M^{D-k-1}\right)$.

We have,

$$
\begin{aligned}
& 3+\alpha=\frac{7}{2}+\beta(D-4) \\
= & \alpha(-k+3)+\frac{1}{2}+k+\beta(D-k-1) .
\end{aligned}
$$

Hence, $\alpha=1$ and $\beta=\frac{1}{2(D-4)}$. $O\left(x^{4}\right)$

The largest error term in this case is $O\left(x^{3} h\right)$, i.e.,

The second case: $O\left(x^{2} h^{2}\right), O\left(x^{\frac{7}{2}} M^{D-4}\right)$ and $O\left(h^{-(k-3)} x^{\frac{1}{2}+k} M^{D-k-1}\right)$

Now,

$$
\begin{aligned}
& 2 \alpha+2=\frac{7}{2}+\beta(D-4) \\
= & \alpha(-k+3)+\frac{1}{2}+k+\beta(D-k-1) .
\end{aligned}
$$

Therefore, $\alpha=\frac{3}{4}+\frac{D-4}{4(D-2)}$ and $\beta=\frac{1}{2 D-4}$.
The dominant error term is $O\left(x^{3} h\right)$, i.e., $O\left(x^{\frac{15}{4}+\frac{1}{4} \frac{D-4}{D-2}}\right)$.

The third case: $O\left(x h^{3}\right), O\left(x^{\frac{7}{2}} M^{D-4}\right)$ and $O\left(h^{-(k-3)} x^{\frac{1}{2}+k} M^{D-k-1}\right)$

We obtain,

$$
\begin{aligned}
& 3 \alpha+1=\frac{7}{2}+\beta(D-4) \\
= & \alpha(-k+3)+\frac{1}{2}+k+\beta(D-k-1) .
\end{aligned}
$$

Thus, $\alpha=\frac{5}{6}+\frac{D-4}{6(D-1)}$ and $\beta=\frac{1}{2 D-2}$.

Once again, the largest error term is $O\left(x^{3} h\right)$, i.e., $O\left(x^{\frac{23}{6}+\frac{1}{6} \frac{D-4}{D-1}}\right)$.

The fourth case: $O\left(h^{4}\right), O\left(x^{\frac{7}{2}} M^{D-4}\right)$ and $O\left(h^{-(k-3)} x^{\frac{1}{2}+k} M^{D-k-1}\right)$

In this case,

$$
\begin{aligned}
& 4 \alpha=\frac{7}{2}+\beta(D-4) \\
= & \alpha(-k+3)+\frac{1}{2}+k+\beta(D-k-1) .
\end{aligned}
$$

It follows that, $\alpha=\frac{7}{8}+\frac{D-4}{8 D}$ and $\beta=\frac{1}{2 D}$.

The largest error term is $O\left(x^{3} h\right)$, i.e., $O\left(x^{\frac{31}{8}+\frac{1}{8} \frac{D-4}{D}}\right)$.

It is easily verified that the optimal error term is $O\left(x^{\frac{15}{4}+\frac{1}{4} \frac{D-4}{D-2}}\right)$, and is achieved for $h=x^{\frac{3}{4}+\frac{D-4}{4(D-2)}}$, $M=x^{\frac{1}{2 D-4}}$.

The sum of $h^{-(k-3)} \Delta_{k-3}^{+} c_{s^{q}}(q, k)$, where $q$ runs over $\{0,1, \ldots, 4\}$, and $s^{q}$ runs over $S_{\mathbb{R}}^{q}, 0<s^{q} \leq \frac{3}{4}$, is

$$
\begin{aligned}
& \sum_{q=0}^{3}(-1)^{q} \sum_{\substack{s^{q} \in S_{\mathbb{R}}^{q} \\
0<s^{q} \leq \frac{3}{4}}} o_{s^{q}}^{q}\left(s^{q}\right)^{-1}\left(s^{q}+1\right)^{-1} \times \\
& \times\left(s^{q}+2\right)^{-1}\left(s^{q}+3\right)^{-1} x^{s^{q}+3}+ \\
& O\left(x^{\frac{11}{4}} h\right) .
\end{aligned}
$$

Namely, $2^{s^{q}+3}$ is $\sum_{j=0}^{+\infty}\left(\begin{array}{c}s^{q}+3 \\ j\end{array}\right)$.

Reasoning as in the previous section, we conclude that the corresponding sum over $q \in\{0,1, \ldots, 4\}$ and $-j \in\{-4,-5, \ldots,-k\}$ is 0 . 
Furthermore, the sum of $h^{-(k-3)} \Delta_{k-3}^{+} c_{-3}(q, k)$, where $q$ runs over $\{0,1, \ldots, 4\}$, is

$$
-\frac{1}{6} \sum_{q=0}^{4}(-1)^{q} \frac{Z_{P, \wedge^{q} \overline{\mathfrak{n}}}^{\prime}\left(-3+\frac{q}{4}\right)}{Z_{P, \wedge^{q} \overline{\mathfrak{n}}}\left(-3+\frac{q}{4}\right)} .
$$

Moreover,

$$
\begin{aligned}
& \sum_{q=0}^{4}(-1)^{q} h^{-(k-3)} \Delta_{k-3}^{+} c_{-2}(q, k)=O(x), \\
& \sum_{q=0}^{4}(-1)^{q} h^{-(k-3)} \Delta_{k-3}^{+} c_{-1}(q, k)=O\left(x^{2} \log x\right), \\
& \sum_{q=0}^{4}(-1)^{q} h^{-(k-3)} \Delta_{k-3}^{+} c_{0}(q, k)=O\left(x^{3} \log x\right) .
\end{aligned}
$$

Note that $h^{-(k-3)} \Delta_{k-3}^{+} c_{s^{q}}(q, k)$ is $o_{s^{q}}^{q}\left(s^{q}\right)^{-1}\left(s^{q}+1\right)^{-1}\left(s^{q}+2\right)^{-1}\left(s^{q}+3\right)^{-1} \tilde{x}_{s^{q}, q, k}^{s^{q}+3}$, when $q$ runs along $\{0,1, \ldots, 4\}$, and $s^{q}$ runs along $S_{\mathbb{R}}^{q},-1<s^{q}<0$, where $\tilde{x}_{s^{q}, q, k}$ belongs to $[x, x+(k-3) h]$.

Thus, the corresponding sum is $O\left(x^{3}\right)$.

Put $h=x^{\frac{3}{4}+\frac{1}{4} \frac{D-4}{D-2}}, M=x^{\frac{1}{2} \frac{1}{D-2}}$.

Having in mind the results derived above, we conclude that $\psi_{3}(x)$ is equal to

$$
\begin{aligned}
& \frac{1}{12} x^{4}+\sum_{q=0}^{3}(-1)^{q} \sum_{\substack{s^{q} \in S_{\mathbb{R}}^{q} \\
0<s^{q} \leq \frac{3}{4}}} o_{s^{q}}^{q}\left(s^{q}\right)^{-1}\left(s^{q}+1\right)^{-1} \times \\
& \times\left(s^{q}+2\right)^{-1}\left(s^{q}+3\right)^{-1} x^{s^{q}+3}+O\left(x^{\frac{15}{4}+\frac{1}{4} \frac{D-4}{D-2}}\right) .
\end{aligned}
$$

Thus, we have proved the following result:

Theorem 2. Let $X_{\Gamma}$ be as above. Then, $\frac{\psi_{3}(x)}{x^{3}}$ is

$$
\frac{1}{12} x+O\left(x^{\frac{3}{4}+\frac{1}{4} \frac{D-4}{D-2}}\right)
$$

as $x \rightarrow+\infty$.

\section{Final remarks}

Some important ideas related to this research are found in: [9], [10], [11] and [22].

The error term $O\left(x^{\frac{1}{2}-\frac{1}{2 D}}\right)$, obtained in the generalized sense, is obviously better than the classical one $O\left(x^{1-\frac{1}{2 D}}\right)$.

\section{References:}

[1] M. Avdispahić and Dž. Gušić , A weighted prime geodesic theorem, Math. Balk. 25, 2011, pp. 463-474.

[2] M. Avdispahić and Dž. Gušić, On the error term in the prime geodesic theorem, Bull. Korean Math. Soc. 49, 2012, pp. 367-372.

[3] M. Avdispahić and Dž. Gušić, On the length spectrum for compact locally symmetric spaces of real rank one, WSEAS Trans. on Math. 16, 2017, pp. 303-321.

[4] M. Avdispahić and Dž. Gušić, Prime geodesic theorem for compact even- dimensional locally symmetric spaces of real rank one, Int. J. Pure Math. 4, 2017, pp. 26-36.

[5] P. Buser, Geometry and Spectra of Compact Riemann Surfaces, Birkhauser Boston Inc, Boston 1992

[6] Y. Cai, Prime geodesic theorem for compact even- dimensional locally symmetric spaces of real rank one, J. Theor. Nom. Bord. 14, 2002, pp. 59-72.

[7] A. Deitmar, Geometric zeta - functions of locally symmetric spaces, Am. J. Math. 122, 2000, pp. 887-926.

[8] A. Deitmar and M. Pavey, A prime geodesic theorem for SL4, Ann. Glob. Anal. Geom. 33, 2008, pp. 161-205.

[9] D. Fried, Analytic torsion and closed geodesics on hyperbolic manifolds, Invent. Math. 84, 1986, pp. 523-540.

[10] D. Fried, Torsion and closed geodesics on complex hyperbolic manifolds, Invent. Math. 91, 1988, pp. 31-51.

[11] Y. Gon and J. Park, The zeta functions of Ruelle and Selberg for hyperbolic manifolds with cusps, Math. Ann. 346, 2010, pp. 719-767.

[12] Dž. Gušić, Prime geodesic theorem for compact even - dimensional locally symmetric Riemannian manifolds of strictly negative sectional curvature, WSEAS Trans. on Math. 17, 2018, pp. 188-196.

[13] Dž. Gušić, A Weighted Generalized Prime Geodesic Theorem, WSEAS Trans. on Math. 17, 2018, pp. 237-251.

[14] Dž. Gušić, On the length spectrum for compact, odd - dimensional, real hyperbolic spaces, WSEAS Trans. on Math. 18, 2019, pp. 211-222.

[15] D. Hejhal, The Selberg trace formula for PSL $(2, \mathbb{R})$. Vol. I. Lecture Notes in Mathematics 548, Springer-Verlag, Berlin-Heidelberg 1976 
[16] D. Hejhal, The Selberg Trace Formula for $\operatorname{PSL}(2, R)$, Springer-Verlag, Berlin 1983

[17] H. Huber, Zur analytischen Theorie hyperbolischer Raumformen und Bewegungsgruppen. II, Math. Annalen 142, 1961, pp. 385-398.

[18] H. Huber, Zur analytischen Theorie hyperbolischer Raumformen und Bewegungsgruppen. II (Nachtrag zu Math. Annalen 142, 385-398, 1961), Math. Annalen 143, 1961, pp. 463-464.

[19] H. Iwaniec, Prime geodesic theorem, J. Reine Angew. Math. 349, 1984, pp. 136-158.

[20] A. W. Knapp, Representation Theory of Semisimple Groups, Princeton University Press, Princeton 1986

[21] S. Koyama, Prime geodesic theorem for arithmetic compact surfaces, Int. Math. Res. Not. 8, 1998, pp. 383-388.

[22] J. Park, Analytic torsion and Ruelle zeta functions for hyperbolic manifolds with cusps, $J$. Func. An. 257, 2009, pp. 1713-1758.
[23] J. Park, Ruelle zeta function and prime geodesic theorem for hyperbolic manifolds with cusps, in: G. van Dijk, M. Wakayama (eds.), Casimir force, Casimir operators and Riemann hypothesis, de Gruyter, Berlin 2010, pp. 89-104.

[24] M. Pavey, Class Numbers of Orders in Quartic Fields, University of Tubingen, Tubingen 2006

[25] B. Randol, On the asymptotic distributon of closed geodesics on compact Riemann surfaces, Trans. Amer. Math. Soc. 233, 1977, pp. 241-247.

[26] K. Soundararajan and M. P. Young, The prime geodesic theorem, J. Reine Angew. Math. 676, 2013, pp. 105-120.

[27] B. Speh, The unitary dual of GL(3,R) and GL(4,R), Math. Ann. 258, 1981, pp. 113-133.

[28] H. Tang, The Generalized Prime Number Theorem for Automorphic L-Functions, Chin. Ann. Math. 3, 2009, pp. 251-260.

[29] N. R. Wallach, On the Selberg trace formula in the case of compact quotient, Bull. Amer. Math. Soc. 82, 1976, pp. 171-195. 